\title{
Lack of research aptitude in medical education
}

Sir,

Students are attracted towards the medical profession to become a doctor and not to be a researcher. According to a recent study there are about 1,00,000 undergraduate medical students in India at a given point of time, out of them only $0.9 \%$ of the students have shown research aptitude. ${ }^{1}$ During their training period of graduation in medical sciences, they are so much burdened with the work load of exams, practicals, ward duties and tutorials. In such an over burdened situation very few of them can think about research. A study had shown that training in research methodology received early in medical school helps students to develop a positive attitude towards research. $^{2}$ So changes in the undergraduate and postgraduate curriculum are required to promote research among medical students.

After graduation, students either go for clinical practice or prepare for Post Graduate Medical Entrance Examination (PGMEE). There are many branches in post graduation studies but these can be divided broadly into two types i.e. clinical, and non clinical branches. Most of the higher ranked students opt for clinical branches as compared to non clinical branches and their choice of branch depend on existing trend as well as the branch by which they can make more money/fame. There are very few students, who will choose any one of the branches on the basis of research orientation. Nowadays the top rankers of PGMEE are opting for the radio-diagnosis and this is not because of their research orientation but the packages and airconditioned life opportunities after completing their PG degree and for this purposes some candidates are ready to pay large amount of money, even a few crores of rupees for a seat allotment of MD (radio-diagnosis) in private Medical Colleges in India. ${ }^{5}$ Most of the Doctors want to do private clinical practice due to more economical benefits attached with it rather than inclining towards the research. To develop research orientation in budding doctors there must be a training programme. The points discussed above, tell us that why we are lacking in research in medical field and raise further few questions as why none of our medical institutes is under even top 200 institutes of the world. ${ }^{6}$

This is the scenario of medical research among the doctors and we need to come out of this situation. Government is doing many things to promote research by different organizations e.g. ICMR, DBT, CSIR etc. In addition, change in the curriculum of UG and PG medical students is must. Further, certain steps can be taken to promote research among medical teachers. Government should mainly invest on those doctors who are working as faculty in medical institutes and plan for them a Compulsory Practical Training Research Workshop (CPTRW) in every speciality. The CPTRW must be absolutely practical; where everything starting from the research hypothesis \& methodology to result \& conclusion should be made understand with practical examples. There must be a practical training programme for handling state-of-art machines/equipments and different basic procedures used during research in relative fields. Then it will change the whole scenario of research just by changing the orientation of mind towards the logical research with vision and expertise. This training termed as investment as these faculties in near future are going to train to their students with right vision and expertise of research, so that culture of satisfying and fruitful research will develop in medical institutions of our country.

Nowadays Medical Council of India (MCI) is conducting Basic Course Workshop in Medical Education Technologies for the medical teachers to make them efficient teacher. In the same way government of India must consider for the CPTRW for medical teachers to make them efficient researcher.

\section{Narendra Kumar, Sarvesh Singh, Rishi Pal, Rahul Kumar* \\ Department of Pharmacology, King George's Medical University, Lucknow, India \\ *Correspondence to: Dr. Rahul Kumar, Email: rahulkgmu@gmail.com}

\section{REFERENCES}

1. Deo MG. Undergraduate medical students' research in India. JPGM;54(3):176-9.

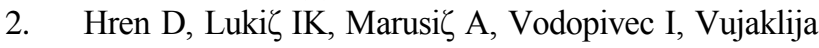
A, Hrabak M, et al. Teaching research methodology in medical schools: Students' attitudes towards and knowledge about science. Med Educ 2004;38:81-6.

3. Ogunyami D, Bazargan M, Norris K, Jones-Quaidoo $\mathrm{S}$, Wolf K, Edelstein R, et al. The development of a mandatory medical thesis in an urban medical school. Teach Learn Med 2005;17:363-9. 
4. Shankar PR, Chandraskekhar TS, Mishra P, Subish P. Initiating and strengthening medical student research: Time to take up the gauntlet. Kath Univ Med J 2006;4:135-8.

5. http://articles.timesofindia.indiatimes.com/2012-0426/indore/31409597_1_mbbs-seat-capitation-medicalcolleges.
6. http://www.thehindu.com/features/education/collegeand-university/no-indian-institute-in-worlds-top-200universities/article3932025.ece.

doi:10.5455/2319-2003.ijbcp20140245

Cite this article as: Kumar N, Singh S, Pal R, Kumar $\mathrm{R}$. Lack of research aptitude in medical education. Int J Basic Clin Pharmacol 2014;3:247-8. 